\title{
Derivatives of self-intersection local times
}

Jay Rosen ${ }^{\star}$

Department of Mathematics

College of Staten Island, CUNY

Staten Island, NY 10314

e-mail: jrosen3@earthlink.net

Summary. We show that the renormalized self-intersection local time $\gamma_{t}(x)$ for both the Brownian motion and symmetric stable process in $R^{1}$ is differentiable in the spatial variable and that $\gamma_{t}^{\prime}(0)$ can be characterized as the continuous process of zero quadratic variation in the decomposition of a natural Dirichlet process. This Dirichlet process is the potential of a random Schwartz distribution. Analogous results for fractional derivatives of self-intersection local times in $R^{1}$ and $R^{2}$ are also discussed.

\section{Introduction}

In their study of the intrinsic Brownian local time sheet and stochastic area integrals for Brownian motion, [14, 15, 16], Rogers and Walsh were led to analyze the functional

$$
A\left(t, B_{t}\right)=\int_{0}^{t} 1_{[0, \infty)}\left(B_{t}-B_{s}\right) \mathrm{d} s
$$

where $B_{t}$ is a 1 -dimensional Brownian motion. They showed that $A\left(t, B_{t}\right)$ is not a semimartingale, and in fact showed that

$$
A\left(t, B_{t}\right)-\int_{0}^{t} L_{s}^{B_{s}} \mathrm{~d} B_{s}
$$

has finite non-zero $4 / 3$-variation. Here $L_{s}^{x}$ is the local time at $x$, which is formally $L_{s}^{x}=\int_{0}^{s} \delta\left(B_{r}-x\right) \mathrm{d} r$, where $\delta(x)$ is Dirac's ' $\delta$-function'. A formal application of Ito's lemma, using $\frac{\mathrm{d}}{\mathrm{d} x} 1_{[0, \infty)}(x)=\delta(x)$ and $\frac{\mathrm{d}^{2}}{\mathrm{~d} x^{2}} 1_{[0, \infty)}(x)=\delta^{\prime}(x)$, yields

$$
A\left(t, B_{t}\right)-\int_{0}^{t} L_{s}^{B_{s}} \mathrm{~d} B_{s}=t+\frac{1}{2} \int_{0}^{t} \int_{0}^{s} \delta^{\prime}\left(B_{s}-B_{r}\right) \mathrm{d} r \mathrm{~d} s
$$

\footnotetext{
* This research was supported, in part, by grants from the National Science Foundation and PSC-CUNY.
} 
which motivates the subject matter of this paper. We study the process which is formally defined as

$$
\gamma_{t}^{\prime}=-\int_{0}^{t} \int_{0}^{s} \delta^{\prime}\left(X_{s}-X_{r}\right) \mathrm{d} r \mathrm{~d} s
$$

where $\delta^{\prime}$ is the derivative of the delta-function, and $X_{t}$ is Brownian motion or, more generally, a symmetric stable process in $R^{1}$. The process $\gamma_{t}^{\prime}$ is related to the self-intersection local time process which is formally defined as

$$
\alpha_{t}=\int_{0}^{t} \int_{0}^{s} \delta\left(X_{s}-X_{r}\right) \mathrm{d} r \mathrm{~d} s
$$

If we set

$$
\alpha_{t}(y)=\int_{0}^{t} \int_{0}^{s} \delta\left(X_{s}-X_{r}-y\right) \mathrm{d} r \mathrm{~d} s
$$

we obtain a 'near intersection' local time, and formally differentiating in $y$ suggests that (4) is the derivative $\left.\frac{\mathrm{d}}{\mathrm{d} y} \alpha_{t}(y)\right|_{y=0}$.

The process $\alpha_{t}$ has not been studied much in one dimension since it can be expressed in terms of the local time $L_{t}^{y}$ of the process $X_{t}: \alpha_{t}=\frac{1}{2} \int\left(L_{t}^{y}\right)^{2} \mathrm{~d} y$ and $\alpha_{t}(y)=\iint_{0}^{t} L_{s}^{x-y} \mathrm{~d}_{s} L_{s}^{x} \mathrm{~d} x$. The fact that $L_{t}^{y}$ is not differentiable in the spatial variable $y$ indicates that the existence of (4) and its identification as a derivative requires some care.

In two dimensions, even for Brownian motion, $\alpha_{t}$ does not exist and must be 'renormalized' by subtracting off a counterterm. This was first done by Varadhan [22], and has been the subject of a large literature, see Dynkin [4], Le Gall [12], Bass and Khoshnevisan [1], Rosen [20, 21]. The resulting renormalized self-intersection local time turns out to be the right tool for the solution of certain "classical" problems such as the asymptotic expansion of the area of the Wiener and stable sausage in the plane and fluctuations of the range of stable random walks. (See Le Gall [11, 10], Le Gall-Rosen [13] and Rosen [19]).

The process $\gamma_{t}^{\prime}$ in $R^{1}$, in a certain sense, is even more singular than selfintersection local time in $R^{2}$, but as we shall see, due to the symmetry properties of $\delta^{\prime}$, there is no need for a counterterm. We begin with a precise definition of $\gamma_{t}^{\prime}$, show that it exists, is the spatial derivative of the renormalized self-intersection local time $\gamma_{t}(x)=\alpha_{t}(x)-E\left(\alpha_{t}(x)\right)$, and has zero quadratic variation. We then show how it can be characterized as the continuous process of zero quadratic variation in the decomposition of a natural Dirichlet process. This Dirichlet process is the potential of a random Schwartz distribution.

Let

$$
\alpha_{t, \epsilon}(y) \stackrel{\text { def }}{=} \int_{0}^{t} \int_{0}^{s} f_{\epsilon}\left(X_{s}-X_{r}-y\right) \mathrm{d} r \mathrm{~d} s
$$

and

$$
\alpha_{t, \epsilon}^{\prime}(y) \stackrel{\text { def }}{=}-\int_{0}^{t} \int_{0}^{s} f_{\epsilon}^{\prime}\left(X_{s}-X_{r}-y\right) \mathrm{d} r \mathrm{~d} s
$$


where $f_{\epsilon}$ is an approximate $\delta$-function at zero, i.e. $f_{\epsilon}(x)=f(x / \epsilon) / \epsilon$ with $f$ a positive, $C^{1}$, even function of $x$ supported in the unit interval with $\int f \mathrm{~d} x=1$. We then define

$$
\begin{gathered}
\alpha_{t}(y)=\lim _{\epsilon \rightarrow 0} \alpha_{t, \epsilon}(y), \quad \alpha_{t}^{\prime}(y)=\lim _{\epsilon \rightarrow 0} \alpha_{t, \epsilon}^{\prime}(y) \\
\gamma_{t}(y)=\lim _{\epsilon \rightarrow 0}\left(\alpha_{t, \epsilon}(y)-E\left(\alpha_{t, \epsilon}(y)\right)\right)
\end{gathered}
$$

and

$$
\gamma_{t}^{\prime}(y)=\lim _{\epsilon \rightarrow 0}\left(\alpha_{t, \epsilon}^{\prime}(y)-E\left(\alpha_{t, \epsilon}^{\prime}(y)\right)\right)
$$

whenever the limit exists. We set $\gamma_{t}^{\prime}=\gamma_{t}^{\prime}(0)$. Let

$$
h_{\beta}(x)= \begin{cases}c(\beta) \operatorname{sgn}(x)|x|^{\beta-2} & \text { if } x \neq 0 \\ 0 & \text { if } x=0\end{cases}
$$

where $c(\beta)=-\pi^{-1} \Gamma(2-\beta) \cos ((1-\beta) \pi / 2)$ if $1<\beta<2$ and $c(2)=-1$. Note that $h_{\beta}(x)$ is not continuous at $x=0$.

Theorem 1. Let $X_{t}$ denote the symmetric stable process of order $\beta>3 / 2$ in $R^{1}$. Then $\alpha_{t, \epsilon}(y)$ and $\alpha_{t, \epsilon}^{\prime}(y)$ converge a.s. and in all $L^{p}$ spaces as $\epsilon \rightarrow 0$ for any $(t, y) \in R_{+} \times R^{1}$.

The following hold almost surely:

1. For any continuous function $g(y)$ we have

$$
\int_{0}^{t} \int_{0}^{s} g\left(X_{s}-X_{r}\right) \mathrm{d} r \mathrm{~d} s=\int g(y) \alpha_{t}(y) \mathrm{d} y .
$$

and if $g \in C^{1}$

$$
\int_{0}^{t} \int_{0}^{s} g^{\prime}\left(X_{s}-X_{r}\right) \mathrm{d} r \mathrm{~d} s=-\int g(y) \alpha_{t}^{\prime}(y) \mathrm{d} y .
$$

2. $\alpha_{t}^{\prime}(y)-h_{\beta}(y) t$ is continuous in $y$.

3. $\left\{\gamma_{t}(y),(t, y) \in R_{+} \times R^{1}\right\}$ and $\left\{\gamma_{t}^{\prime}(y),(t, y) \in R_{+} \times R^{1}\right\}$ are continuous and $\gamma_{t}(y)$ is differentiable in $y$ with $\gamma_{t}^{\prime}(y)=\frac{\mathrm{d}}{\mathrm{d} y} \gamma_{t}(y)$.

4. $\alpha_{t}^{\prime}(0)=\gamma_{t}^{\prime}(0)$.

We see from (13) and (14) that $\alpha_{t}^{\prime}(y)$ is the distributional derivative of $\alpha_{t}(y)$. However, we see from 2. that $\alpha_{t}^{\prime}(y)$ is not continuous at $y=0$ so that these equations do not allow us to characterize $\alpha_{t}^{\prime}(0)$.

For any function $g_{t}$ and any sequence $\tau=\left\{\tau_{n}\right\}$ of partitions $\tau_{n}=\{0=$ $\left.t_{0}<t_{n, 1}<\cdots<t_{n, n}=T\right\}$ of $[0, T]$, with mesh size $\left|\tau_{n}\right|=\max _{i}\left|t_{n, i}-t_{n, i-1}\right|$ going to 0 , we set

$$
V_{p}(g ; \tau)=\lim _{n \rightarrow \infty} \sum_{i=1}^{n}\left|g_{t_{n, i}}-g_{t_{n, i-1}}\right|^{p}
$$


whenever it exists.

In [16], Rogers and Walsh show that for Brownian motion $V_{4 / 3}\left(\gamma^{\prime} ; \tau\right)$ is a finite non-zero constant, independent of $\tau$. Let $\beta^{\prime}$ denote the usual conjugate exponent to $\beta$, i.e. $\frac{1}{\beta}+\frac{1}{\beta^{\prime}}=1$.

Theorem 2. Let $X$ be a symmetric stable process of order $\beta>3 / 2$ in $R^{1}$. Then $V_{p}\left(\gamma^{\prime} ; \tau\right)=0$ for any $\tau$ and any $p>\frac{2 \beta^{\prime}}{3}$.

Note that for Brownian motion this shows that $V_{p}\left(\gamma^{\prime} ; \tau\right)=0$ for any $p>4 / 3$. We conjecture that for the symmetric stable process of order $\beta>3 / 2$ in $R^{1}$ we have that $V_{\frac{2 \beta^{\prime}}{3}}\left(\gamma^{\prime} ; \tau\right)$ is a finite non-zero constant, independent of $\tau$.

We now obtain an intrinsic characterization for $\gamma_{t}^{\prime}$, which doesn't involve limits. The key idea is that $\gamma_{t}^{\prime}$ has zero quadratic variation. In the case of renormalized intersection local time $\gamma_{t}$ for Brownian motion in the plane this was observed by Bertoin, [2] and extended by us in [21].

Recall that a continuous adapted process $Z_{t}$ is said to have zero quadratic variation, if for each $T>0$ and any sequence of partitions $\tau_{n}=\left\{0=t_{0}<\right.$ $\left.t_{1}<\cdots<t_{n}=T\right\}$ of $[0, T]$, with mesh size $\left|\tau_{n}\right|=\max _{i}\left|t_{i}-t_{i-1}\right|$ going to 0

$$
\lim _{n \rightarrow \infty} E\left(\sum_{t_{i} \in \tau_{n}}\left(Z_{t_{i}}-Z_{t_{i-1}}\right)^{2}\right)=0 .
$$

Föllmer [7] has coined the term "Dirichlet process" to refer to any process which can be written as the sum of a martingale and a process of zero quadratic variation. It is important to note that such a decomposition is unique. The class of Dirichlet processes is much wider than the class of semimartingales.

We use $Y_{t}$ to denote our stable process $X_{t}$ killed at an independent exponential time $\lambda$. In the following theorem $\gamma_{t}^{\prime}$ will be defined for the process $Y_{t}$ in place of $X_{t}$.

Let us begin with a special case of the Doob-Meyer decomposition for semimartingales. Let $L_{t}^{\mu}$ denotes the continuous additive functional of $X_{t}$ with Revuz measure $\mu$. Using the additivity of $L_{t}^{\mu}$ and the Markov property we have

$$
E^{x}\left(L_{\lambda}^{\mu} \mid \mathcal{F}_{t}\right)=L_{t \wedge \lambda}^{\mu}+U^{1} \mu\left(Y_{t}\right)
$$

where $\mathcal{F}_{t}=\sigma\left(Y_{s}, s \leq t\right)$. Equivalently, $U^{1} \mu\left(Y_{t}\right)=M_{t}-L_{t \wedge \lambda}^{\mu}$ where $M_{t}=$ $E^{x}\left(L_{\lambda}^{\mu} \mid \mathcal{F}_{t}\right)$ is a martingale. This is the Doob-Meyer decomposition for the potential $U^{1} \mu\left(Y_{t}\right)$. We will show that $\gamma_{t}^{\prime}$ arises in a similar decomposition for the potential of a random Schwartz distribution. This new potential will no longer be a semimartingale but a Dirichlet process, and $\gamma_{t}^{\prime}$ will correspond to the process of zero quadratic variation in the decomposition of this Dirichlet process.

Let $F_{t}$ be the random additive distribution-valued process defined by

$$
F_{t}(g)=-\lim _{\epsilon \rightarrow 0} \int_{0}^{t} \int g\left(y+Y_{r}\right) f_{\epsilon}^{\prime}(y) \mathrm{d} y \mathrm{~d} r
$$


whenever the limit exists. It follows by integration by parts that for all $g \in$ $C_{0}^{\infty}\left(R^{1}\right)$

$$
F_{t}(g)=\int_{0}^{t} g^{\prime}\left(Y_{r}\right) \mathrm{d} r
$$

With this definition it is natural to set

$$
U^{1} F_{t}(x)=-\lim _{\epsilon \rightarrow 0} \int_{0}^{t} \int u^{1}\left(x-\left(y+Y_{r}\right)\right) f_{\epsilon}^{\prime}(y) \mathrm{d} y \mathrm{~d} r
$$

whenever the limit exists.

Note that formally

$$
U^{1} F_{t}(x)=-\int_{0}^{t} \frac{\mathrm{d} u^{1}}{\mathrm{~d} x}\left(x-Y_{s}\right) \mathrm{d} s .
$$

We have the following analogue of the Doob-Meyer decomposition.

Theorem 3. Let $Y$ be a symmetric stable process of order $\beta>3 / 2$ in $R^{1}$, killed at an independent exponential time $\lambda$. Then $\gamma_{t}^{\prime}$ is continuous a.s. with zero quadratic variation and

$$
U^{1} F_{t}\left(Y_{t}\right)=M_{t}-\gamma_{t}^{\prime}
$$

where $M_{t}$ is the martingale $E^{x}\left(\gamma_{\infty}^{\prime} \mid \mathcal{F}_{t}\right)$.

In view of Theorem 3 we can characterize the renormalized intersection local time $\gamma_{t}^{\prime}$ as the continuous process of zero quadratic variation in the decomposition of the random potential $U^{1} F_{t}\left(Y_{t}\right)$ which is formally $-\int_{0}^{t} \frac{d u^{1}}{\mathrm{~d} x}\left(Y_{t}-Y_{s}\right) \mathrm{d} s$.

\subsection{Fractional derivatives}

All our results can be extended to fractional derivatives. There are in fact several natural candidates for the fractional derivative of order $0<\rho<1$ in $R^{d}$ : $g^{(\rho)}(x)=(2 \pi)^{-d} \int \mathrm{e}^{i p x} w(p) \widehat{g}(p) \mathrm{d} p$ with $w(p)$ positively homogeneous of index $\rho$, i.e. $w(\lambda p)=|\lambda|^{p} w(p)$ for all $\lambda>0$. Our results can be extended for any such $w(p)$, but for simplicity we work with symmetric fractional derivatives: $w(-p)=-w(p)$ which allows us to avoid introducing counterterms. In one dimension this determines $w(p)$ up to a constant factor: $w(p)=\operatorname{sgn}(p)|p|^{\rho}$. Then we study

$$
\gamma_{t}^{(\rho)}=-\int_{0}^{t} \int_{0}^{s} \delta^{(\rho)}\left(X_{s}-X_{r}\right) \mathrm{d} r \mathrm{~d} s
$$

More precisely, let

$$
\alpha_{t, \epsilon}^{(\rho)}(y) \stackrel{\text { def }}{=}-\int_{0}^{t} \int_{0}^{s} f_{\epsilon}^{(\rho)}\left(X_{s}-X_{r}-y\right) \mathrm{d} r \mathrm{~d} s,
$$




$$
\alpha_{t}^{(\rho)}(y)=\lim _{\epsilon \rightarrow 0} \alpha_{t, \epsilon}^{(\rho)}(y)
$$

and

$$
\gamma_{t}^{(\rho)}(y)=\lim _{\epsilon \rightarrow 0}\left(\alpha_{t, \epsilon}^{(\rho)}(y)-E\left(\alpha_{t, \epsilon}^{(\rho)}(y)\right)\right)
$$

whenever the limit exists. We set $\gamma_{t}^{(\rho)}=\gamma_{t}^{(\rho)}(0)$.

Theorem 4. Let $X_{t}$ denote the symmetric stable process of order $\beta>1 \mathrm{~V}$ $(\rho+1 / 2)$ in $R^{1}$. Then $\alpha_{t, \epsilon}^{(\rho)}(y)$ converges a.s. and in all $L^{p}$ spaces as $\epsilon \rightarrow 0$ for any $(t, y) \in R_{+} \times R^{1}$.

The following hold almost surely:

1. For any $C^{\rho}$ function $g(x)$ we have

$$
\int_{0}^{t} \int_{0}^{s} g^{(\rho)}\left(X_{s}-X_{r}\right) \mathrm{d} r \mathrm{~d} s=-\int g(y) \alpha_{t}^{(\rho)}(y) \mathrm{d} y .
$$

2. $\alpha_{t}^{(\rho)}(y)-h_{\beta+1-\rho}(y) t$ is continuous in $y$.

3. $\left\{\gamma_{t}^{(\rho)}(y),(t, y) \in R_{+} \times R^{1}\right\}$ is a.s. continuous and $\gamma_{t}^{(\rho)}(y)$ is the derivative of order $\rho$ in $y$ of $\gamma_{t}(y)$.

4. $\alpha_{t}^{(\rho)}(0)=\gamma_{t}^{(\rho)}(0)$.

5. $V_{p}\left(\gamma^{(\rho)} ; \tau\right)=0$ for any $\tau$ and any $p>\frac{2 \beta}{3 \beta-1-2 \rho}$.

Once again, we use $Y_{t}$ to denote our $R^{1}$ valued Lévy process $X_{t}$ killed at an independent exponential time $\lambda$ and in the following theorem $\gamma_{t}^{(\rho)}$ will be defined for the process $Y_{t}$ in place of $X_{t}$. Let now $\Phi_{t}$ be the random additive distribution-valued process defined by

$$
\Phi_{t}(g)=-\lim _{\epsilon \rightarrow 0} \int_{0}^{t} \int g\left(y+Y_{r}\right) f_{\epsilon}^{(\rho)}(y) \mathrm{d} y \mathrm{~d} r
$$

whenever the limit exists. It is easy to check that for all $g \in C_{0}^{\infty}\left(R^{1}\right)$

$$
\Phi_{t}(g)=\int_{0}^{t} g^{(\rho)}\left(Y_{r}\right) \mathrm{d} r
$$

With this definition it is natural to set

$$
U^{1} \Phi_{t}(x)=-\lim _{\epsilon \rightarrow 0} \int_{0}^{t} \int u^{1}\left(x-\left(y+Y_{r}\right)\right) f_{\epsilon}^{(\rho)}(y) \mathrm{d} y \mathrm{~d} r
$$

whenever the limit exists.

Note that formally

$$
U^{1} \Phi_{t}(x)=-\int_{0}^{t}\left(u^{1}\right)^{(\rho)}\left(x-Y_{r}\right) \mathrm{d} r
$$

We have the following analogue of the Doob-Meyer decomposition. 
Theorem 5. Let $Y$ be a symmetric stable process of order $\beta>\rho+1 / 2$ in $R^{1}$, killed at an independent exponential time $\lambda$. Then $\gamma_{t}^{(\rho)}$ is continuous a.s. with zero quadratic variation and

$$
U^{1} \Phi_{t}\left(Y_{t}\right)=M_{t}-\gamma_{t}^{(\rho)}
$$

where $M_{t}$ is the martingale $E^{x}\left(\gamma_{\lambda}^{(\rho)} \mid \mathcal{F}_{t}\right)$.

We leave to the interested reader the task of formulating analogous results for fractional derivatives of renormalized self-intersection local times in the plane.

This paper is organized as follows. In section 2 we prove Theorem 1 and in section 3 prove Theorem 2. Section 4 contains the short proof of Theorem 3 . The proofs of Theorems 4 and 5 are similar and left to the reader.

Acknowledgement. I would like to thank J. Walsh for some very helpful conversations.

\section{Existence of $\alpha_{t}^{\prime}(x)$}

Proof of Theorem 1. For any $x \in R^{1}$ and bounded Borel set $B \subseteq R_{+}^{2}$ let

$$
\alpha_{\epsilon}^{\prime}(x, B)=-\int_{B} \int f_{\epsilon}^{\prime}\left(X_{s}-X_{r}-x\right) \mathrm{d} r \mathrm{~d} s .
$$

$\alpha_{\epsilon}^{\prime}(x, B)$ is clearly continuous in all parameters as long as $\epsilon>0$. We use $|B|$ to denote the Lebesgue measure of $B \subseteq R_{+}^{2}$. For any random variable $Y$ we set $\{Y\}_{0}=Y-E(Y)$.

The following Lemma will be proven at the end of this section.

Lemma 1. Let $X$ be the symmetric stable process of index $\beta>3 / 2$ in $R^{1}$. Then for some $\zeta>0$

$$
\left|E\left(\left\{\alpha_{\epsilon}^{\prime}(x, B)-\alpha_{\epsilon^{\prime}}^{\prime}\left(x^{\prime}, B\right)\right\}_{0}^{j}\right)\right| \leq c_{0}(\zeta, j)\left|(\epsilon, x)-\left(\epsilon^{\prime}, x^{\prime}\right)\right|^{j \zeta}
$$

and

$$
\left|E\left(\left\{\alpha_{\epsilon}^{\prime}(x, B)\right\}_{0}^{j}\right)\right| \leq c_{0}(\zeta, j)|B|^{j \zeta}
$$

for all $j \in Z_{+}, \epsilon, \epsilon^{\prime} \in(0,1], x, x^{\prime} \in R^{1}$ and all Borel sets $B \subseteq A_{1}^{1}=$ : $[0,1 / 2] \times[1 / 2,1]$.

Let

$$
A_{k}^{n}=\left[(2 k-2) 2^{-n},(2 k-1) 2^{-n}\right] \times\left[(2 k-1) 2^{-n},(2 k) 2^{-n}\right] .
$$

Using the scaling $X_{\lambda t} \stackrel{\mathcal{L}}{=} \lambda^{1 / \beta} X_{t}$ and $f_{\lambda \epsilon}^{\prime}(x)=\frac{1}{\lambda^{2}} f_{\epsilon}^{\prime}(x / \lambda)$ we have 


$$
\alpha_{\epsilon}^{\prime}(x, B) \stackrel{\mathcal{L}}{=} 2^{-n(2-2 / \beta)} \alpha_{2^{n / \beta} \epsilon}^{\prime}\left(2^{n / \beta} x, 2^{n} B\right)
$$

so that from (34) and (35) we have that for all Borel sets $B \subseteq A_{k}^{n+1}$

$\left|E\left(\left\{\alpha_{\epsilon}^{\prime}(x, B)-\alpha_{\epsilon^{\prime}}^{\prime}\left(x^{\prime}, B\right)\right\}_{0}^{j}\right)\right| \leq c_{0}(\zeta, j) 2^{-n j(2-2 / \beta-\zeta / \beta)}\left|(\epsilon, x)-\left(\epsilon^{\prime}, x^{\prime}\right)\right|^{\zeta j}$

and

$$
\left|E\left(\left\{\alpha_{\epsilon}^{\prime}(x, B)\right\}_{0}^{j}\right)\right| \leq c_{0}(\zeta, j) 2^{-n j(2-2 / \beta-2 \zeta)}|B|^{\zeta j} .
$$

For any $B$ let

$$
\gamma_{\epsilon}^{\prime}(x, B)=\left\{\alpha_{\epsilon}^{\prime}(x, B)\right\}_{0}
$$

Following Le Gall [12] we write

$$
\gamma_{\epsilon}^{\prime}(x, B)=\sum_{n, k} \gamma_{\epsilon}^{\prime}\left(x, B \cap A_{k}^{n}\right)
$$

for any $B \subseteq\{0 \leq r \leq s \leq 1\}$. Using (38) and (39) together with independence we then have, (see Prop. 3.5.2 of [8]),

$$
\begin{aligned}
& \left|E\left(\left\{\sum_{k=1}^{2^{n}} \gamma_{\epsilon}^{\prime}\left(x, B \cap A_{k}^{n+1}\right)-\gamma_{\epsilon^{\prime}}^{\prime}\left(x^{\prime}, B \cap A_{k}^{n+1}\right)\right\}^{j}\right)\right| \\
& \leq c_{0}(\zeta, j) 2^{n j / 2} 2^{-n j(2-2 / \beta-\zeta / \beta)}\left|(\epsilon, x)-\left(\epsilon^{\prime}, x^{\prime}\right)\right|^{\zeta j}
\end{aligned}
$$

and

$$
\left|E\left(\left\{\sum_{k=1}^{2^{n}} \gamma_{\epsilon}^{\prime}\left(x, B \cap A_{k}^{n+1}\right)\right\}^{j}\right)\right| \leq c_{0}(\zeta, j) 2^{n j / 2} 2^{-n j(2-2 / \beta-2 \zeta)}|B|^{\zeta j}
$$

so that

$$
\left\|\gamma_{\epsilon}^{\prime}(x, B)-\gamma_{\epsilon^{\prime}}^{\prime}\left(x^{\prime}, B\right)\right\|_{j} \leq c\left|(\epsilon, x)-\left(\epsilon^{\prime}, x^{\prime}\right)\right|^{\zeta}
$$

and

$$
\left\|\gamma_{\epsilon}^{\prime}(x, B)\right\|_{j} \leq c|B|^{\zeta}
$$

if we choose $\zeta>0$ so that $1 / 2-2+2 / \beta+\zeta(2+1 / \beta)<0$. This is possible for $\beta>4 / 3$ (and we are assuming that $\beta>3 / 2$ ).

Let $B_{t}=\{0 \leq r \leq s \leq t\}$ and set $\gamma_{\epsilon, t}^{\prime}(x)=: \gamma_{\epsilon}^{\prime}\left(x, B_{t}\right)$. If $t, t^{\prime} \leq M<\infty$ then $\left|B_{t}-B_{t^{\prime}}\right| \leq M\left|t-t^{\prime}\right|$ so that by (45) for some $c<\infty$

$$
\left\|\gamma_{\epsilon, t}^{\prime}(x)-\gamma_{\epsilon, t^{\prime}}^{\prime}(x)\right\|_{j} \leq c\left|t-t^{\prime}\right|^{\zeta}
$$

and combined with (44) this shows that for some $\zeta>0$

$$
\left\|\gamma_{\epsilon, t}^{\prime}(x)-\gamma_{\epsilon^{\prime}, t^{\prime}}^{\prime}\left(x^{\prime}\right)\right\|_{j} \leq c\left|(\epsilon, x, t)-\left(\epsilon^{\prime}, x^{\prime}, t^{\prime}\right)\right|^{\zeta}
$$

Kolmogorov's lemma then shows that locally 


$$
\left|\gamma_{\epsilon, t}^{\prime}(x)-\gamma_{\epsilon^{\prime}, t^{\prime}}^{\prime}\left(x^{\prime}\right)\right| \leq c_{\omega}\left|(\epsilon, x, t)-\left(\epsilon^{\prime}, x^{\prime}, t^{\prime}\right)\right|^{\zeta^{\prime}}, \quad \epsilon, \epsilon^{\prime}>0
$$

for some $\zeta^{\prime}>0$, which assures us of a locally uniform and hence continuous limit

$$
\gamma_{t}^{\prime}(x)=\lim _{\epsilon \rightarrow 0} \gamma_{\epsilon, t}^{\prime}(x)
$$

The next Lemma is proven in section 5 .

Lemma 2. For the symmetric stable process of index $\beta>1$ we can find a continuous function $h(x)$ with $h(0)=0$ such that for each $x$

$$
\lim _{\epsilon \rightarrow 0} E\left(\alpha_{\epsilon, t}^{\prime}(x)\right)-h(x)= \begin{cases}c(\beta) \operatorname{sgn}(x)|x|^{\beta-2} t & \text { if } x \neq 0 \\ 0 & \text { if } x=0\end{cases}
$$

where $c(\beta)=-2 \Gamma(2-\beta) \cos ((1-\beta) \pi / 2)$ if $1<\beta<2$ and $c(2)=-1$. Furthermore, (50) converges locally uniformly in $x$ away from 0 and locally in $L^{1}$.

Using this Lemma and the locally uniform convergence (49) we see that

$$
\alpha_{t}^{\prime}(x)=\lim _{\epsilon \rightarrow 0} \alpha_{\epsilon, t}^{\prime}(x)
$$

exists for all $x, t$, and $\alpha_{t}^{\prime}(0)=\gamma_{t}^{\prime}(0)$. Furthermore the convergence is locally uniform in $x$ away from 0 and locally in $L^{1}$.

A similar and simpler analysis shows that locally

$$
\left|\gamma_{\epsilon, t}(x)-\gamma_{\epsilon^{\prime}, t^{\prime}}\left(x^{\prime}\right)\right| \leq c_{\omega}\left|(\epsilon, x, t)-\left(\epsilon^{\prime}, x^{\prime}, t^{\prime}\right)\right|^{\zeta^{\prime}}, \quad \epsilon, \epsilon^{\prime}>0
$$

for some $\zeta^{\prime}>0$, which assures us of a locally uniform and hence continuous limit

$$
\gamma_{t}(x)=\lim _{\epsilon \rightarrow 0} \gamma_{\epsilon, t}(x)
$$

Using the bound $p_{s}(y) \leq c / s^{1 / \beta}$ we can check that $\int_{0}^{t} \int_{0}^{s} p_{s-r}(y) \mathrm{d} r \mathrm{~d} s$ is bounded and continuous in $y$ and that uniformly in $x$

$$
\begin{aligned}
\lim _{\epsilon \rightarrow 0} E\left(\alpha_{\epsilon, t}(x)\right) & =\lim _{\epsilon \rightarrow 0} \int_{0}^{t} \int_{0}^{s}\left(\int f_{\epsilon}(y-x) p_{s-r}(y) \mathrm{d} y\right) \mathrm{d} r \mathrm{~d} s \\
& =\lim _{\epsilon \rightarrow 0} \int f_{\epsilon}(y-x)\left(\int_{0}^{t} \int_{0}^{s} p_{s-r}(y) \mathrm{d} r \mathrm{~d} s\right) \mathrm{d} y \\
& =\int_{0}^{t} \int_{0}^{s} p_{s-r}(x) \mathrm{d} r \mathrm{~d} s
\end{aligned}
$$

Together with the locally uniform convergence of (53) we see that

$$
\alpha_{t}(x)=\lim _{\epsilon \rightarrow 0} \alpha_{\epsilon, t}(x) .
$$

locally uniformly. 
Now let $g(x)$ be a $C^{1}$ function with compact support. The locally $L^{1}$ convergence in (51) and the fact that $\left\{X_{s} ; 0 \leq s \leq t\right\}$ is bounded a.s. shows that

$$
\begin{aligned}
\int g(x) \alpha_{t}^{\prime}(x) \mathrm{d} x & =\lim _{\epsilon \rightarrow 0} \int g(x) \alpha_{\epsilon, t}^{\prime}(x) \mathrm{d} x \\
& =-\lim _{\epsilon \rightarrow 0} \int g(x)\left(\int_{0}^{t} \int_{0}^{s} f_{\epsilon}^{\prime}\left(X_{s}-X_{r}-x\right) \mathrm{d} r \mathrm{~d} s\right) \mathrm{d} x \\
& =-\lim _{\epsilon \rightarrow 0} \int_{0}^{t} \int_{0}^{s}\left(\int g^{\prime}(x) f_{\epsilon}\left(X_{s}-X_{r}-x\right) \mathrm{d} x\right) \mathrm{d} r \mathrm{~d} s \\
& =-\lim _{\epsilon \rightarrow 0} \int_{0}^{t} \int_{0}^{s} f_{\epsilon} * g^{\prime}\left(X_{s}-X_{r}\right) \mathrm{d} r \mathrm{~d} s \\
& =-\int_{0}^{t} \int_{0}^{s} g^{\prime}\left(X_{s}-X_{r}\right) \mathrm{d} r \mathrm{~d} s .
\end{aligned}
$$

Since the path $\left\{X_{s} ; 0 \leq s \leq t\right\}$ is bounded a.s. we have that $\alpha_{t}^{\prime}(x)$ has compact support a.s. so that (56) holds for all $C^{1}$ functions $g(x)$.

Similarly, using the locally uniform convergence (55) we see that for any continuous function $h(x)$ we have

$$
\int h(x) \alpha_{t}(x) \mathrm{d} x=\int_{0}^{t} \int_{0}^{s} h\left(X_{s}-X_{r}\right) \mathrm{d} r \mathrm{~d} s .
$$

Therefore

$$
\int g(x) \alpha_{t}^{\prime}(x) \mathrm{d} x=-\int g^{\prime}(x) \alpha_{t}(x) \mathrm{d} x
$$

holds for all $C^{1}$ functions $g(x)$.

It is clear that $\frac{\mathrm{d}}{\mathrm{d} x} \gamma_{\epsilon, t}(x)=\gamma_{\epsilon, t}^{\prime}(x)$ for any $\epsilon>0$ and hence

$$
\gamma_{\epsilon, t}(x)=\gamma_{\epsilon, t}(y)+\int_{y}^{x} \gamma_{\epsilon, t}^{\prime}(z) \mathrm{d} z
$$

The locally uniform convergence shown above then implies that

$$
\gamma_{t}(x)=\gamma_{t}(y)+\int_{y}^{x} \gamma_{t}^{\prime}(z) \mathrm{d} z
$$

and therefore $\frac{\mathrm{d}}{\mathrm{d} x} \gamma_{t}(x)=\gamma_{t}^{\prime}(x)$. This completes the proof of Theorem 1 .

Proof of Lemma 1. We begin by showing how to find a bound

$$
\left|E\left(\left(\alpha_{\epsilon}^{\prime}(x, B)\right)^{j}\right)\right| \leq c^{j}
$$

uniform in $\epsilon \in(0,1], x \in R^{1}$ and $B \subseteq A_{1}^{1}$.

We use 


$$
f_{\epsilon}^{\prime}(x)=\frac{i}{2 \pi} \int \mathrm{e}^{i p x} p \widehat{f}(\epsilon p) \mathrm{d} p
$$

and independence to write that

$$
\begin{aligned}
& E\left(\left(\alpha_{\epsilon}^{\prime}(x, B)\right)^{j}\right) \\
& =\frac{1}{(2 \pi i)^{j}} \iint_{B^{j}} \exp \left(-i x \sum_{k=1}^{j} p_{k}\right) E\left(\exp \left(\sum_{k=1}^{j} i p_{k}\left(X_{s_{k}}-X_{r_{k}}\right)\right)\right) \\
& =\frac{1}{(2 \pi i)^{j}} \iint_{B^{j}} \exp \left(-i x \sum_{k=1}^{j} p_{k} \widehat{f}\left(\epsilon p_{k}\right) \mathrm{d} s_{k} \mathrm{~d} r_{k} \mathrm{~d} p_{k}\right. \\
& E\left(\exp \left(\sum_{k=1}^{j} i p_{k}\left(X_{s_{k}}-X_{1 / 2}\right)\right)\right) \prod_{k=1}^{j} p_{k} \widehat{f}\left(\epsilon p_{k}\right) \mathrm{d} s_{k} \mathrm{~d} r_{k} \mathrm{~d} p_{k} .
\end{aligned}
$$

We write

$$
\sum_{k=1}^{j} p_{k}\left(X_{1 / 2}-X_{r_{k}}\right)=\sum_{k=1}^{j} v_{k}\left(X_{t_{k+1}}-X_{t_{k}}\right)
$$

where the $t_{1}, \ldots, t_{j}$ are the $r_{i}$ 's relabeled so that $t_{1} \leq t_{2} \leq \cdots \leq t_{j} \leq t_{j+1} \stackrel{\text { def }}{=}$ $1 / 2$ and $v_{i}=\sum_{l: r_{l} \leq t_{i}} p_{l}$ so that the $v_{i}$ 's span $R^{j}$. Similarly we rewrite

$$
\sum_{k=1}^{j} p_{k}\left(X_{s_{k}}-X_{1 / 2}\right)=\sum_{k=1}^{j} v_{k}^{\prime}\left(X_{t_{k}^{\prime}}-X_{t_{k-1}^{\prime}}\right)
$$

with $t_{0}^{\prime} \stackrel{\text { def }}{=} 1 / 2$. Then using (63) and independence we have

$$
\begin{aligned}
& E\left(\left(\alpha_{\epsilon}^{\prime}(x, B)\right)^{j}\right) \\
& =\frac{1}{(2 \pi i)^{j}} \iint_{B^{j}} \exp \left(-i x \sum_{k=1}^{j} p_{k}\right) \exp \left(-\sum_{k=1}^{j}\left|v_{k}\right|^{\beta}\left(t_{k+1}^{\prime}-t_{k}^{\prime}\right)\right) \\
& \quad \exp \left(-\sum_{k=1}^{j}\left|v_{k}^{\prime}\right|^{\beta}\left(t_{k}-t_{k-1}\right)\right) \prod_{k=1}^{j} p_{k} \widehat{f}\left(\epsilon p_{k}\right) \mathrm{d} s_{k} \mathrm{~d} r_{k} \mathrm{~d} p_{k} .
\end{aligned}
$$

Using this and the simple bound

$$
\int_{0}^{1} \mathrm{e}^{-t|v|^{\beta}} \mathrm{d} t \leq \frac{c}{1+|v|^{\beta}}
$$

we have the uniform bound 


$$
\begin{aligned}
\left|E\left(\left(\alpha_{\epsilon}^{\prime}(x, B)\right)^{j}\right)\right| & \leq c^{j} \int \prod \frac{1}{1+\left|v_{k}\right|^{\beta}} \prod \frac{1}{1+\left|v_{k}^{\prime}\right|^{\beta}} \prod_{k=1}^{j}\left|p_{k}\right| \mathrm{d} p_{k} \\
& \leq c^{j}\left\|\prod \frac{\left|p_{k}\right|^{1 / 2}}{1+\left|v_{k}\right|^{\beta}}\right\|_{2}\left\|\prod \frac{\left|p_{k}\right|^{1 / 2}}{1+\left|v_{k}^{\prime}\right|^{\beta}}\right\|_{2} .
\end{aligned}
$$

Since $v_{i}=\sum_{l: r_{l} \leq t_{i}} p_{l}$, we see that each $p_{k}$ can be represented as the difference $p_{k}=v_{i}-v_{i-1}$ for some $i$, and each $v_{i}$ appears in the representation of at most two $p_{k}$ 's. Thus

$$
\begin{aligned}
\left\|\prod \frac{\left|p_{k}\right|^{1 / 2}}{1+\left|v_{k}\right|^{\beta}}\right\|_{2}^{2} & =\int \prod \frac{\left|p_{k}\right|}{1+\left|v_{k}\right|^{2 \beta}} \mathrm{d} p_{k} \\
& \leq c^{j} \int \prod \frac{1+\left|v_{k}\right|+\left|v_{k}\right|^{2}}{1+\left|v_{k}\right|^{2 \beta}} \mathrm{d} v_{k}
\end{aligned}
$$

which is bounded if $\beta>3 / 2$.

We can now establish (34). To handle the variation in $x$ we replace $(66)$ by

$$
\begin{aligned}
& E\left(\left(\alpha_{\epsilon}^{\prime}(x, B)-\alpha_{\epsilon}^{\prime}\left(x^{\prime}, B\right)\right)^{j}\right) \\
& =\frac{1}{(2 \pi i)^{j}} \iint_{B^{j}}\left(\prod_{k=1}^{j}\left\{\exp \left(-i p_{k} x\right)-\exp \left(-i p_{k} x^{\prime}\right)\right\}\right) \\
& \quad \exp \left(-\sum_{k=1}^{j}\left|v_{k}\right|^{\beta}\left(t_{k+1}^{\prime}-t_{k}^{\prime}\right)\right) \exp \left(-\sum_{k=1}^{j}\left|v_{k}^{\prime}\right|^{\beta}\left(t_{k}-t_{k-1}\right)\right) \\
& \prod_{k=1}^{j} p_{k} \widehat{f}\left(\epsilon p_{k}\right) \mathrm{d} s_{k} \mathrm{~d} r_{k} \mathrm{~d} p_{k}
\end{aligned}
$$

and use the bound

$$
\left|\exp \left(-i p_{k} x\right)-\exp \left(-i p_{k} x^{\prime}\right)\right| \leq C\left|p_{k}\right|^{\zeta}\left|x-x^{\prime}\right|^{\zeta}
$$

for any $0 \leq \zeta \leq 1$

Similarly to handle the variation in $\epsilon$ we replace (66) by

$$
\begin{aligned}
& E\left(\left(\alpha_{\epsilon}^{\prime}(x, B)-\alpha_{\epsilon^{\prime}}^{\prime}(x, B)\right)^{j}\right) \\
& =\frac{1}{(2 \pi i)^{j}} \iint_{B^{j}} \exp \left(-i x \sum_{k=1}^{j} p_{k}\right) \exp \left(-\sum_{k=1}^{j}\left|v_{k}\right|^{\beta}\left(t_{k+1}^{\prime}-t_{k}^{\prime}\right)\right) \\
& \quad \exp \left(-\sum_{k=1}^{j}\left|v_{k}^{\prime}\right|^{\beta}\left(t_{k}-t_{k-1}\right)\right) \prod_{k=1}^{j} p_{k}\left(\widehat{f}\left(\epsilon p_{k}\right)-\widehat{f}\left(\epsilon^{\prime} p_{k}\right)\right) \mathrm{d} s_{k} \mathrm{~d} r_{k} \mathrm{~d} p_{k}
\end{aligned}
$$

and use the bound 


$$
\left|\widehat{f}\left(\epsilon p_{k}\right)-\widehat{f}\left(\epsilon^{\prime} p_{k}\right)\right| \leq C\left|p_{k}\right|^{\zeta}\left|\epsilon-\epsilon^{\prime}\right|^{\zeta}
$$

for any $0 \leq \zeta \leq 1$. Since (70)-(72) hold also when $j=1$, we obtain (34).

To prove (35) we first apply Holder's inequality to (66):

$$
\begin{aligned}
& E\left(\left(\alpha_{\epsilon}^{\prime}(x, B)\right)^{j}\right) \\
& =\frac{1}{(2 \pi i)^{j}} \iint_{B^{j}} \exp \left(-i x \sum_{k=1}^{j} p_{k}\right) \exp \left(-\sum_{k=1}^{j}\left|v_{k}\right|^{\beta}\left(t_{k+1}^{\prime}-t_{k}^{\prime}\right)\right) \\
& \quad \exp \left(-\sum_{k=1}^{j}\left|v_{k}^{\prime}\right|^{\beta}\left(t_{k}-t_{k-1}\right)\right) \prod_{k=1}^{j} p_{k} \widehat{f}\left(\epsilon p_{k}\right) \mathrm{d} s_{k} \mathrm{~d} r_{k} \mathrm{~d} p_{k} \\
& \leq c^{j}|B|^{j / a} \int\left(\int_{B^{j}} \exp \left(-a^{\prime} \sum_{k=1}^{j}\left|v_{k}\right|^{\beta}\left(t_{k+1}^{\prime}-t_{k}^{\prime}\right)\right)\right. \\
& \left.\exp \left(-a^{\prime} \sum_{k=1}^{j}\left|v_{k}^{\prime}\right|^{\beta}\left(t_{k}-t_{k-1}\right)\right) \prod_{k=1}^{j} \mathrm{~d} s_{k} \mathrm{~d} r_{k}\right)^{1 / a^{\prime}} \prod_{k=1}^{j}\left|p_{k}\right| \mathrm{d} p_{k}
\end{aligned}
$$

for any $1 / a+1 / a^{\prime}=1$. The last integral can be bounded as before if $a^{\prime}$ is chosen close to 1. As in the proof of (34), this completes the proof of (35) and therefore of Lemma 1.

\section{3 p-variation of $\gamma_{t}^{\prime}$}

Proof of Theorem 2. Since we know that $\alpha_{t, \epsilon}^{\prime} \rightarrow \gamma_{t}^{\prime}$ in $L^{2}$, we have

$$
\begin{aligned}
& E\left(\left(\gamma_{t}^{\prime}-\gamma_{t^{\prime}}^{\prime}\right)^{2}\right) \\
& =\lim _{\epsilon \rightarrow 0} E\left(\left(\alpha_{t, \epsilon}^{\prime}-\alpha_{t^{\prime}, \epsilon}^{\prime}\right)^{2}\right) \\
& =\lim _{\epsilon \rightarrow 0} E\left(\left(\int_{t^{\prime}}^{t} \int_{0}^{s} f_{\epsilon}^{\prime}\left(X_{s}-X_{r}\right) \mathrm{d} r \mathrm{~d} s\right)^{2}\right) \\
& =\lim _{\epsilon \rightarrow 0} \iint_{\left\{\begin{array}{c}
0 \leq s_{1} \leq s_{2} \leq s_{3} \leq s_{4} \\
t^{\prime} \leq s_{3} \leq t ; t^{\prime} \leq s_{4} \leq t
\end{array}\right\}} E\left(\exp \left(-i p\left(X_{s_{2}}-X_{s_{1}}\right)-i(p+q)\left(X_{s_{3}}-X_{s_{2}}\right)\right)\right. \\
& \left.+\lim _{\epsilon \rightarrow 0} \iint_{\left\{\begin{array}{c}
0 \leq s_{1} \leq s_{2} \leq s_{3} \leq s_{4} \\
t^{\prime} \leq s_{3} \leq t ; t^{\prime} \leq s_{4} \leq t
\end{array}\right\}} E\left(-i q\left(X_{s_{4}}-X_{s_{3}}\right)\right)\right) \prod_{k=1}^{4} \mathrm{~d} s_{k} p q \widehat{f}(\epsilon p) \widehat{f}(\epsilon q) \mathrm{d} p \mathrm{~d} q \\
& =\lim _{\epsilon \rightarrow 0} \iint_{\left\{\begin{array}{c}
0 \leq s_{1} \leq s_{2} \leq s_{3} \leq s_{4} \\
t^{\prime} \leq s_{3} \leq t ; t^{\prime} \leq s_{4} \leq t
\end{array}\right\}} \mathrm{exp}^{-\left(-i p\left(x_{2}-s_{1}\right)|p|^{\beta}-\left(x_{3}-s_{2}\right)|p+q|^{\beta}-\left(s_{4}-s_{3}\right)|q|^{\beta}\right.} X_{\left.\left.\left.s_{1}\right)-i(p+q)\left(X_{s_{3}}-X_{s_{2}}\right)\right)\right)} \prod_{k=1}^{4} \mathrm{~d} s_{k} p q \widehat{f}(\epsilon p) \widehat{f}(\epsilon q) \mathrm{d} p \mathrm{~d} q
\end{aligned}
$$




$$
\begin{aligned}
& \prod_{k=1}^{4} \mathrm{~d} s_{k} p q \widehat{f}(\epsilon p) \widehat{f}(\epsilon q) \mathrm{d} p \mathrm{~d} q \\
& +\lim _{\epsilon \rightarrow 0} \iint\left\{\begin{array}{c}
0 \leq s_{1} \leq s_{2} \leq s_{3} \leq s_{4} \\
t^{\prime} \leq s_{3} \leq t ; t^{\prime} \leq s_{4} \leq t
\end{array}\right\} \mathrm{e}^{-\left(s_{2}-s_{1}\right)|p|^{\beta}-\left(s_{3}-s_{2}\right)|p+q|^{\beta}-\left(s_{4}-s_{3}\right)|p|^{\beta}} \\
& \prod_{k=1}^{4} \mathrm{~d} s_{k} p q \widehat{f}(\epsilon p) \widehat{f}(\epsilon q) \mathrm{d} p \mathrm{~d} q
\end{aligned}
$$

Consider the first summand on the right hand side of (75). It is bounded by

$$
\begin{gathered}
\iint\left\{\begin{array}{c}
t^{\prime} \leq r_{1}+r_{2}+r_{3} \leq t \\
t^{\prime} \leq r_{1}+r_{2}+r_{3}+r_{4} \leq t
\end{array}\right\} \\
\quad \leq C\left(t-t^{\prime}\right)^{1+a} \int \frac{1}{1+|p|^{\beta}} \frac{1}{1+|p+q|^{\beta}} \frac{1}{1+|q|^{(1-a) \beta}}|p||q| \mathrm{d} p \mathrm{~d} q
\end{gathered}
$$

where we first integrated with respect to $\mathrm{d} r_{4}$ using Hölder's inequality with $0 \leq a \leq 1$

$$
\int_{t^{\prime}-\left(r_{1}+r_{2}+r_{3}\right)}^{t-\left(r_{1}+r_{2}+r_{3}\right)} \mathrm{e}^{-r_{4}|q|^{\beta}} \mathrm{d} r_{4} \leq\left(t-t^{\prime}\right)^{a}\left(\int_{0}^{t} \mathrm{e}^{-r_{4}|q|^{\beta} /(1-a)} \mathrm{d} r_{4}\right)^{(1-a)},
$$

then with respect to $\mathrm{d} r_{1}$ using $\int_{t^{\prime}-\left(r_{2}+r_{3}\right)}^{t-\left(r_{2}\right)} \mathrm{d} r_{1} \leq t-t^{\prime}$, and finally the $\mathrm{d} r_{2}$, $\mathrm{d} r_{3}$ integrals are bounded using (67). It is easily seen that (76) is bounded as long as $\beta-1+(1-a) \beta-1>1$, i.e. $a<2-3 / \beta$.

Now consider the second summand on the right hand side of (75). An attempt to use a bound similar to (76) where we bound $p q$ by $|p q|$ would be fatal. Rather, we first observe that $\int \mathrm{e}^{-\left(s_{3}-s_{2}\right)|q|^{\beta}} q \mathrm{~d} q=0$ and use this to rewrite the second summand on the right hand side of (75) as

$$
\begin{aligned}
& \lim _{\epsilon \rightarrow 0} \iint\left\{\begin{array}{c}
0 \leq s_{1} \leq s_{2} \leq s_{3} \leq s_{4} \\
t^{\prime} \leq s_{3} \leq t ; t^{\prime} \leq s_{4} \leq t
\end{array}\right\} \\
& \quad \mathrm{e}^{-\left(s_{2}-s_{1}\right)|p|^{\beta}-\left(s_{4}-s_{3}\right)|p|^{\beta}} \\
& \left.\mathrm{e}^{-\left(s_{3}-s_{2}\right)|p+q|^{\beta}}-\mathrm{e}^{-\left(s_{3}-s_{2}\right)|p|^{\beta}} \mathrm{e}^{-\left(s_{3}-s_{2}\right)|q|^{\beta}}\right\} \prod_{k=1}^{4} \mathrm{~d} s_{k} p q \widehat{f}(\epsilon p) \widehat{f}(\epsilon q) \mathrm{d} p \mathrm{~d} q .
\end{aligned}
$$

Now we bound this as in (76) by

$$
\begin{aligned}
& \iint_{\left\{\begin{array}{c}
t^{\prime} \leq r_{1}+r_{2}+r_{3} \leq t \\
t^{\prime} \leq r_{1}+r_{2}+r_{3}+r_{4} \leq t
\end{array}\right\}^{-r_{2}|p|^{\beta}-r_{4}|p|^{\beta}}}\left|\mathrm{e}^{-r_{3}|p+q|^{\beta}}-\mathrm{e}^{-r_{3}|p|^{\beta}} \mathrm{e}^{-r_{3}|q|^{\beta}}\right| \prod_{k=1}^{4} \mathrm{~d} r_{k}|p||q| \mathrm{d} p \mathrm{~d} q \\
& \leq C\left(t-t^{\prime}\right)^{1+a} \int \frac{1}{1+|p|^{(2-a) \beta}}\left|\frac{1}{1+|p+q|^{\beta}}-\frac{1}{1+|p|^{\beta}+|q|^{\beta}}\right||p||q| \mathrm{d} p \mathrm{~d} q .
\end{aligned}
$$


Here we proceeded as in (76) except that for the $\mathrm{d} r_{3}$ integral we used

$$
\int \mathrm{e}^{-r}\left|\mathrm{e}^{-r|p+q|^{\beta}}-\mathrm{e}^{-r\left(|p|^{\beta}+|q|^{\beta}\right)}\right| \mathrm{d} r \leq\left|\frac{1}{1+|p+q|^{\beta}}-\frac{1}{1+|p|^{\beta}+|q|^{\beta}}\right|
$$

by arguing seperately depending on whether or not $|p|^{\beta}+|q|^{\beta}>|p+q|^{\beta}$. We claim that once again the integral on the right hand side of (79) is finite whenever $\beta-1+(1-a) \beta-1>1$, i.e. $a<2-3 / \beta$. This is clear in the region where $|q| \leq 2|p|$ since we can use the bound $\frac{1}{1+|p|^{(2-a) \beta}} \leq \frac{1}{1+|p|^{\beta}} \frac{c}{1+|q|^{(1-a) \beta}}$. If, however, $|q|>2|p|$, we can use the bound

$$
\begin{aligned}
\left|\frac{1}{1+|p+q|^{\beta}}-\frac{1}{1+|p|^{\beta}+|q|^{\beta}}\right| & =\frac{|l| p+\left.q\right|^{\beta}-|q|^{\beta}-|p|^{\beta} \mid}{\left(1+|p+q|^{\beta}\right)\left(1+|p|^{\beta}+|q|^{\beta}\right)} \\
& \leq \frac{c|p||q|^{\beta-1}+|p|^{\beta}}{\left(1+|p+q|^{\beta}\right)\left(1+|p|^{\beta}+|q|^{\beta}\right)} \\
& \leq \frac{c|p||q|^{\beta-1}}{\left(1+|q|^{\beta}\right)^{2}} .
\end{aligned}
$$

This allows us to bound the resulting integral from the right hand side of (79) by

$$
c \int \frac{|p|^{2}}{1+|p|^{(2-a) \beta}} \frac{1}{\left(1+|q|^{\beta}\right)} \mathrm{d} p \mathrm{~d} q
$$

which leads to the same result as before.

Finally, for $h \leq 2$ we have

$$
E\left(\left(\gamma_{t}^{\prime}-\gamma_{t^{\prime}}^{\prime}\right)^{h}\right) \leq\left\{E\left(\left(\gamma_{t}^{\prime}-\gamma_{t^{\prime}}^{\prime}\right)^{2}\right)\right\}^{h / 2} \leq C\left(t-t^{\prime}\right)^{(1+a) h / 2} .
$$

We will have h-variation 0 when $(1+a) h / 2>1$ for some $a<2-3 / \beta$, i.e. $(3-3 / \beta) h / 2>1$. Thus we will have h-variation 0 when $h>(2 / 3) \beta^{\prime}$. This completes the proof of Theorem 2.

\section{The Doob-Meyer decomposition}

Proof of Theorem 3. It is easy to check that the proofs of Theorems 1 and 2 go through with $X$ replaced by $Y$ and in that case

$$
\gamma_{\infty}^{\prime}=\lim _{\epsilon \rightarrow 0} \gamma_{\epsilon, \infty}^{\prime} \quad \text { and } \quad \gamma_{t}^{\prime}=\lim _{\epsilon \rightarrow 0} \gamma_{\epsilon, t}^{\prime}
$$

converge a.s. and in all $L^{p}$ spaces. Let

$$
U^{1} F_{\epsilon, t}(y)=-\int_{0}^{t} \int u^{1}\left(x-\left(y-Y_{r}\right)\right) f_{\epsilon}^{\prime}(x) \mathrm{d} x \mathrm{~d} r .
$$

The proof of Theorem 3 then follows from (84) and the next Lemma. 
Lemma 3. Let $\left\{Y_{s} ; s \in R_{+}^{1}\right\}$ be the exponentially killed symmetric stable process of index $\beta>3 / 2$ in $R^{1}$. Then for any $\epsilon>0$

$$
U^{1} F_{\epsilon, t}\left(Y_{t}\right)=E^{x}\left(\gamma_{\epsilon, \infty}^{\prime} \mid \mathcal{F}_{t}\right)-\gamma_{\epsilon, t}^{\prime}
$$

Proof of Lemma 3. We have

$$
\gamma_{\epsilon, t}^{\prime}=-\left\{\int_{0}^{t} \int_{0}^{s} f_{\epsilon}^{\prime}\left(Y_{s}-Y_{r}\right) \mathrm{d} r \mathrm{~d} s\right\}
$$

so that

$$
\begin{aligned}
\gamma_{\epsilon, \infty}^{\prime}= & -\left\{\int_{0}^{\infty} \int_{0}^{s} f_{\epsilon}^{\prime}\left(Y_{s}-Y_{r}\right) \mathrm{d} r \mathrm{~d} s\right\} \\
= & \gamma_{\epsilon, t}^{\prime}-\left\{\int_{t}^{\infty} \int_{t}^{s} f_{\epsilon}^{\prime}\left(Y_{s}-Y_{r}\right) \mathrm{d} r \mathrm{~d} s\right\} \\
& -\left\{\int_{t}^{\infty} \int_{0}^{t} f_{\epsilon}^{\prime}\left(Y_{s}-Y_{r}\right) \mathrm{d} r \mathrm{~d} s\right\} .
\end{aligned}
$$

Now, using the fact that $\left\{Y_{s} ; s \in R_{+}^{1}\right\}$ has independent increments

$$
\begin{aligned}
& E\left(\left\{\int_{t}^{\infty} \int_{t}^{s} f_{\epsilon}^{\prime}\left(Y_{s}-Y_{r}\right) \mathrm{d} r \mathrm{~d} s\right\} \mid \mathcal{F}_{t}\right) \\
& =E\left(\int_{t}^{\infty} \int_{t}^{s} f_{\epsilon}^{\prime}\left(Y_{s}-Y_{r}\right) \mathrm{d} r \mathrm{~d} s \mid \mathcal{F}_{t}\right)-E\left(\int_{t}^{\infty} \int_{t}^{s} f_{\epsilon}^{\prime}\left(Y_{s}-Y_{r}\right) \mathrm{d} r \mathrm{~d} s\right)=0
\end{aligned}
$$

and

$$
\begin{aligned}
& E\left(\left\{\int_{t}^{\infty} \int_{0}^{t} f_{\epsilon}^{\prime}\left(Y_{s}-Y_{r}\right) \mathrm{d} r \mathrm{~d} s\right\} \mid \mathcal{F}_{t}\right) \\
& \quad=E\left(\int_{t}^{\infty} \int_{0}^{t} f_{\epsilon}^{\prime}\left(Y_{s}-Y_{r}\right) \mathrm{d} r \mathrm{~d} s \mid \mathcal{F}_{t}\right)-E\left(\int_{t}^{\infty} \int_{0}^{t} f_{\epsilon}^{\prime}\left(Y_{s}-Y_{r}\right) \mathrm{d} r \mathrm{~d} s\right) .
\end{aligned}
$$

We have

$$
\begin{aligned}
E\left(\int_{t}^{\infty} \int_{0}^{t} f_{\epsilon}^{\prime}\left(Y_{s}-Y_{r}\right) \mathrm{d} r \mathrm{~d} s \mid \mathcal{F}_{t}\right) & \\
= & E\left(\int_{t}^{\infty} \int_{0}^{t} f_{\epsilon}^{\prime}\left(\left(Y_{s}-Y_{t}\right)+\left(Y_{t}-Y_{r}\right)\right) \mathrm{d} r \mathrm{~d} s \mid \mathcal{F}_{t}\right) \\
& =\bar{E}\left(\int_{0}^{\infty} \int_{0}^{t} f_{\epsilon}^{\prime}\left(\bar{Y}_{s}+\left(Y_{t}-Y_{r}\right)\right) \mathrm{d} r \mathrm{~d} s\right)
\end{aligned}
$$

where $\left\{\bar{Y}_{s} ; s \in R_{+}^{1}\right\}$ is an independent copy of $\left\{Y_{s} ; s \in R_{+}^{1}\right\}$ and $\bar{E}$ denotes expectation with respect to $\left\{\bar{Y}_{s} ; s \in R_{+}^{1}\right\}$. Hence 


$$
\begin{aligned}
E\left(\int_{t}^{\infty} \int_{0}^{t} f_{\epsilon}^{\prime}\left(Y_{s}-Y_{r}\right) \mathrm{d} r \mathrm{~d} s \mid \mathcal{F}_{t}\right) & =\int_{0}^{t} \int f_{\epsilon}^{\prime}\left(x+Y_{t}-Y_{r}\right) u^{1}(x) \mathrm{d} x \mathrm{~d} r \\
& =\int_{0}^{t} \int u^{1}\left(x-\left(Y_{t}-Y_{r}\right)\right) f_{\epsilon}^{\prime}(x) \mathrm{d} x \mathrm{~d} r
\end{aligned}
$$

The same sort of argument shows that

$$
E\left(\int_{t}^{\infty} \int_{0}^{t} f_{\epsilon}^{\prime}\left(Y_{s}-Y_{r}\right) \mathrm{d} r \mathrm{~d} s\right)=\int_{0}^{t} \int f_{\epsilon}^{\prime}(x+y) u^{1}(x) p_{r}(y) \mathrm{d} x \mathrm{~d} y \mathrm{~d} r
$$

which is zero by symmetry. This concludes the proof of Lemma 3 .

\section{Proof of Lemma 2}

Proof of Lemma 2. We have

$$
E\left(\alpha_{\epsilon, t}^{\prime}(x)\right)=-\int_{0}^{t} \int_{0}^{s}\left(\int f_{\epsilon}^{\prime}(y-x) p_{s-r}(y) \mathrm{d} y\right) \mathrm{d} r \mathrm{~d} s
$$

We first consider the case $1<\beta<2$. Using the fact that $f_{\epsilon}^{\prime}$ is an odd fuction, Plancherel and then Fubini

$$
\begin{aligned}
-\int_{0}^{t} \int_{0}^{s}\left(\int f_{\epsilon}^{\prime}(y-\right. & \left.x) p_{s-r}(y) \mathrm{d} y\right) \mathrm{d} r \mathrm{~d} s \\
= & i(2 \pi)^{-1} \int_{0}^{t} \int_{0}^{s}\left(\int \mathrm{e}^{i p x} p \widehat{f}(\epsilon p) \mathrm{e}^{-(s-r)|p|^{\beta}} \mathrm{d} p\right) \mathrm{d} r \mathrm{~d} s \\
& =i(2 \pi)^{-1} \int \mathrm{e}^{i p x} p \widehat{f}(\epsilon p)\left(\int_{0}^{t} \int_{0}^{s} \mathrm{e}^{-(s-r)|p|^{\beta}} \mathrm{d} r \mathrm{~d} s\right) \mathrm{d} p
\end{aligned}
$$

and

$$
\begin{aligned}
\int_{0}^{t} \int_{0}^{s} & \mathrm{e}^{-(s-r)|p|^{\beta}} \mathrm{d} r \mathrm{~d} s \\
& =\int_{0}^{t} \int_{r}^{t} \mathrm{e}^{-(s-r)|p|^{\beta}} \mathrm{d} s \mathrm{~d} r=\int_{0}^{t}\left(\int_{0}^{t-r} \mathrm{e}^{-s|p|^{\beta}} \mathrm{d} s\right) \mathrm{d} r \\
& =\int_{0}^{t} \int_{0}^{r} \mathrm{e}^{-s|p|^{\beta}} \mathrm{d} s \mathrm{~d} r=t \int_{0}^{\infty} \mathrm{e}^{-s|p|^{\beta}} \mathrm{d} s-\int_{0}^{t} \int_{r}^{\infty} \mathrm{e}^{-s|p|^{\beta}} \mathrm{d} s \mathrm{~d} r \\
& =t|p|^{-\beta}-\int_{0}^{t} \mathrm{e}^{-r|p|^{\beta}}|p|^{-\beta} \mathrm{d} r=t|p|^{-\beta}-\left(1-\mathrm{e}^{-t|p|^{\beta}}\right)|p|^{-2 \beta}
\end{aligned}
$$

Hence

$$
\begin{aligned}
E\left(\alpha_{\epsilon, t}^{\prime}(x)\right)= & i(2 \pi)^{-1} t \int \mathrm{e}^{i p x} \operatorname{sgn}(p)|p|^{1-\beta} \widehat{f}(\epsilon p) \mathrm{d} p \\
& -i(2 \pi)^{-1} \int \mathrm{e}^{i p x} \operatorname{sgn}(p)\left(1-\mathrm{e}^{-t|p|^{\beta}}\right)|p|^{1-2 \beta} \widehat{f}(\epsilon p) \mathrm{d} p .
\end{aligned}
$$


It is easily checked that $\left(1-\mathrm{e}^{-t|p|^{\beta}}\right)|p|^{1-2 \beta} \in L^{1}\left(R^{1}\right)$ so that the last term converges uniformly as $\epsilon \rightarrow 0$ to a continuous limit. On the other hand, it follows from [9, formula (13), page 173] that

$$
\begin{aligned}
& i(2 \pi)^{-1} \int \mathrm{e}^{i p x} \operatorname{sgn}(p)|p|^{1-\beta} \widehat{f}(\epsilon p) \mathrm{d} p \\
& \quad=-\pi^{-1} \Gamma(2-\beta) \cos ((1-\beta) \pi / 2) \int \operatorname{sgn}(y)|y|^{\beta-2} f_{\epsilon}(y-x) \mathrm{d} y .
\end{aligned}
$$

Since $\operatorname{sgn}(y)|y|^{\beta-2}$ is locally in $L^{1}$ and continuous away from 0 , this last term converges locally uniformly away from 0 and locally in $L^{1}$ to $c(\beta) \operatorname{sgn}(x)|x|^{\beta-2}$. On the other hand, when $x=0,(94)$ is 0 by symmetry. This completes the proof of Lemma 2 for $\beta \neq 2$.

For Brownian motion, we proceed differently. We first write

$$
\begin{aligned}
&-\int_{0}^{t} \int_{0}^{s}\left(\int f_{\epsilon}^{\prime}(y-x) p_{s-r}(y) \mathrm{d} y\right) \mathrm{d} r \mathrm{~d} s \\
&=\int_{0}^{t} \int_{0}^{s}\left(\int f_{\epsilon}(y-x) p_{s-r}^{\prime}(y) \mathrm{d} y\right) \mathrm{d} r \mathrm{~d} s .
\end{aligned}
$$

We have $p_{s}^{\prime}(y)=\frac{-1}{(2 \pi)^{1 / 2} s^{3 / 2}} y \mathrm{e}^{-y^{2} / 2 s}$ so that, for $y \neq 0,\left\{\left|p_{s}^{\prime}(y)\right| ; s \geq 0\right\}$ is the density of $T_{|y|}$, the first hitting time of $|y|$ for Brownian motion. Hence

$$
\int_{0}^{\infty}\left|p_{s}^{\prime}(y)\right| \mathrm{d} s=1, \quad y \neq 0 .
$$

This justifies the use of Fubini

$$
\begin{aligned}
\int_{0}^{t} \int_{0}^{s}\left(\int f_{\epsilon}(y-x) p_{s-r}^{\prime}(y) \mathrm{d} y\right) \mathrm{d} r \mathrm{~d} s & \\
& =\int f_{\epsilon}(y-x)\left(\int_{0}^{t} \int_{0}^{s} p_{s-r}^{\prime}(y) \mathrm{d} r \mathrm{~d} s\right) \mathrm{d} y
\end{aligned}
$$

and then, just as in (96)

$$
\int_{0}^{t} \int_{0}^{s} p_{s-r}^{\prime}(y) \mathrm{d} r \mathrm{~d} s=t \int_{0}^{\infty} p_{s}^{\prime}(y) \mathrm{d} s-\int_{0}^{t} \int_{r}^{\infty} p_{s}^{\prime}(y) \mathrm{d} s \mathrm{~d} r, \quad y \neq 0 .
$$

Just as in (100) we see that the first integral is $-\operatorname{sgn}(y)$ for $y \neq 0$. Now $\left|p_{s}^{\prime}(y)\right| \leq \frac{1}{(2 \pi)^{1 / 2} s^{3 / 2}}|y|$, so that by the dominated convergence theorem the second integral is continuous in $y$. As before, when $y=0$, the left hand side of (99) is 0 by symmetry. Lemma 2 now follows. 


\section{References}

1. R. Bass and D. Khoshnevisan, Intersection local times and Tanaka formulas, Ann. Inst. H. Poincaré Prob. Stat. 29 (1993), 419-452.

2. J. Bertoin, Applications des processus de Dirichlet aux temps locaux et temps locaux d'intersection d'un mouvement Brownien, Prob. Theory Related Fields 80 (1989), 433-460.

3. R. Blumenthal and R. Getoor, Some theorems on stable proceses, Trans. Amer. Math. Soc. 95 (1960), 261-273.

4. E. B. Dynkin, Self-intersection gauge for random walks and for Brownian motion, Ann. Probab. 16 (1988), 1-57.

5. P. Fitzsimmons and T. Salisbury, Capacity and energy for multiparameter Markov processes, Ann. Inst. H. Poincaré Prob. Stat. 25 (1989), 325-350.

6. P. Fitzsimmons and R. Getoor, Limit theorems and variation properties for fractional derivatives of the local time of a stable process, Ann. Inst. H. Poincaré Prob. Stat. 28 (1992), 311-333.

7. H. Föllmer, Dirichlet processes, Proceedings Durham 1980, Lecture Notes Math, vol. 851, Springer-Verlag, Berlin, 1981, 476-478.

8. A. Garsia, Topics in Almost Everywhere Convergence, Markham Publishing Company, Chicago (1970).

9. I. M. Gelfand and G. E. Shilov, Generalized Functions, Vol. 1, Academic Press, N. Y. (1964).

10. J.-F. Le Gall, Propriétés d'intersection des marches aléatoires, I, Comm. Math. Phys. 104 (1986), 471-507.

11. , Fluctuation results for the Wiener sausage, Ann. Probab. 16 (1988), 991-1018.

12. S_ Some properties of planar Brownian motion, École d'Été de Probabilités de St. Flour XX, 1990, Lecture Notes in Mathematics, vol. 1527, SpringerVerlag, Berlin, 1992

13. J.-F. Le Gall and J. Rosen, The range of stable random walks, Ann. Probab. 19 (1991), 650-705.

14. L C. G. Rogers and J. B. Walsh, Local time and stochastic area integrals, Ann. Probab. 19 (1991), 457-482.

15. L C. G. Rogers and J. B. Walsh, The intrinsic local time sheet of Brownian motion, Prob. Theory Related Fields 80 (1991), 433-460.

16. L C. G. Rogers and J. B. Walsh, $A\left(t, B_{t}\right)$ is not a semimartingale, Seminar on Stochastic Processes, Birkhauser, Boston. (1991), 457-482.

17. J. Rosen, Joint continuity and a Doob-Meyer type decomposition for renormalized intersection local times, Ann. Inst. H. Poincaré Prob. Stat. 35 (1999), 143176.

18. _ Continuity and singularity of the intersection local time of stable processes in $\mathbf{R}^{2}$, Ann. Probab. 16 (1988), 75-79.

19. _ The asymptotics of stable sausages in the plane, Ann. Probab. 20 (1992), 29-60.

20. Joint continuity of renormalized intersection local times, Ann. Inst. H. Poincaré Prob. Stat. 32 (1996), 671-700.

21. _ Dirichlet processes and an intrinsic characterization of renormalized intersection local times, Ann. Inst. H. Poincaré Prob. Stat. 37 (2001), 403-420.

22. S. R. S. Varadhan, Appendix to Euclidian quantum field theory by K. Symanzyk, Local Quantum Theory (R. Jost, ed.), Academic Press, 1969. 